Content may change prior to man publication in an issue or the journal. 10 cite the paper please use the dor provided on the digital Library page. 


\title{
Co-optimizing Distribution Network Adequacy and Security by Simultaneous Utilization of Network Reconfiguration and Distributed Energy Resources
}

ISSN $1751-8644$ doi: 0000000000 www.ietdl.org

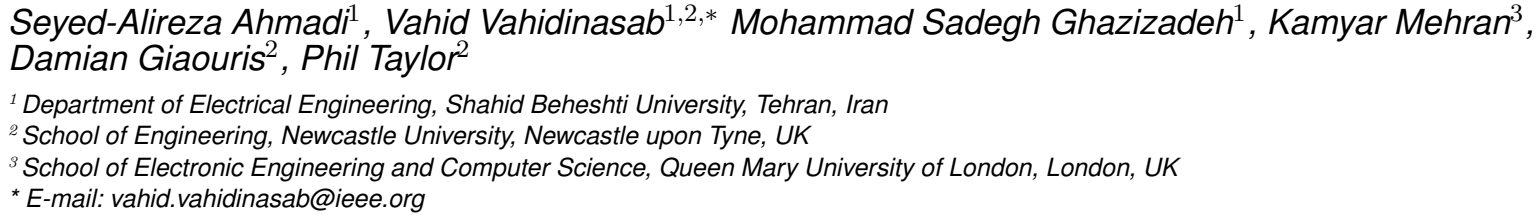

Seyed-Alireza Ahmadi ${ }^{1}$, Vahid Vahidinasab ${ }^{1,2, *}$ Mohammad Sadegh Ghazizadeh ${ }^{1}, K_{\text {Kamyar Mehran }}^{3}$, Damian Giaouris ${ }^{2}$, Phil Taylor ${ }^{2}$

${ }^{1}$ Department of Electrical Engineering, Shahid Beheshti University, Tehran, Iran

${ }^{2}$ School of Engineering, Newcastle University, Newcastle upon Tyne, UK

${ }^{3}$ School of Electronic Engineering and Computer Science, Queen Mary University of London, London, UK

*E-mail: vahid.vahidinasab@ieee.org

\begin{abstract}
With the evolution of smart grids, penetration of distributed energy resources (DERs) in the distribution networks has become ever increasing problem. To improve network reliability, the complexity of the two important aspects of adequacy and security must be well assessed. There is a trade-off between adequacy of DERs, and the distribution network security, i.e. improving the adequacy can reduce the security. In this paper, enhancement of the distribution network adequacy and security (DNAS) is proposed. In this regard, capacity of simultaneous reconfiguration and DERs sizing are utilized to improve the adequacy and security of an active distribution networks. In the reconfiguration process, graph theory concept is adopted to implement a fast reconfiguration method. Since DERs are active, a combined bus and line security index is used to overcome security concerns of their existence. The IEEE 33-bus distribution network as a widely used standard test system in reconfiguration studies, and a practical 83-bus distribution network of Taiwan Power Company (TPC) which is a part of a real distribution network, are used to test the performance of the proposed method. The simulation results demonstrate the performance of the proposed framework.
\end{abstract}

\section{Nomenclature}

Abbreviations

The abbreviations used in this paper are listed below and sorted in alphabetical order for quick reference.
ABC Artificial Bee Colony
AENS Average energy not supplied
AI Adequacy index
BSI Bus voltage stability index
CAIDI Coustomer average interruption duration index
DER Distributed energy resource
DNAS Distribution network adequacy and security
ENS Energy not supplied
AENS Average Energy not supplied
HBBO Human behavior-based optimization
LCU Line capacity usage
LSI Line stability index
MAIDI Momentary average interruption duration index
OVSI Overall voltage stability index
SAIDI System average interruption duration index
SAIFI System average interruption frequency index

MAIFI Momentary average interruption frequency index

\section{Other Symbols}

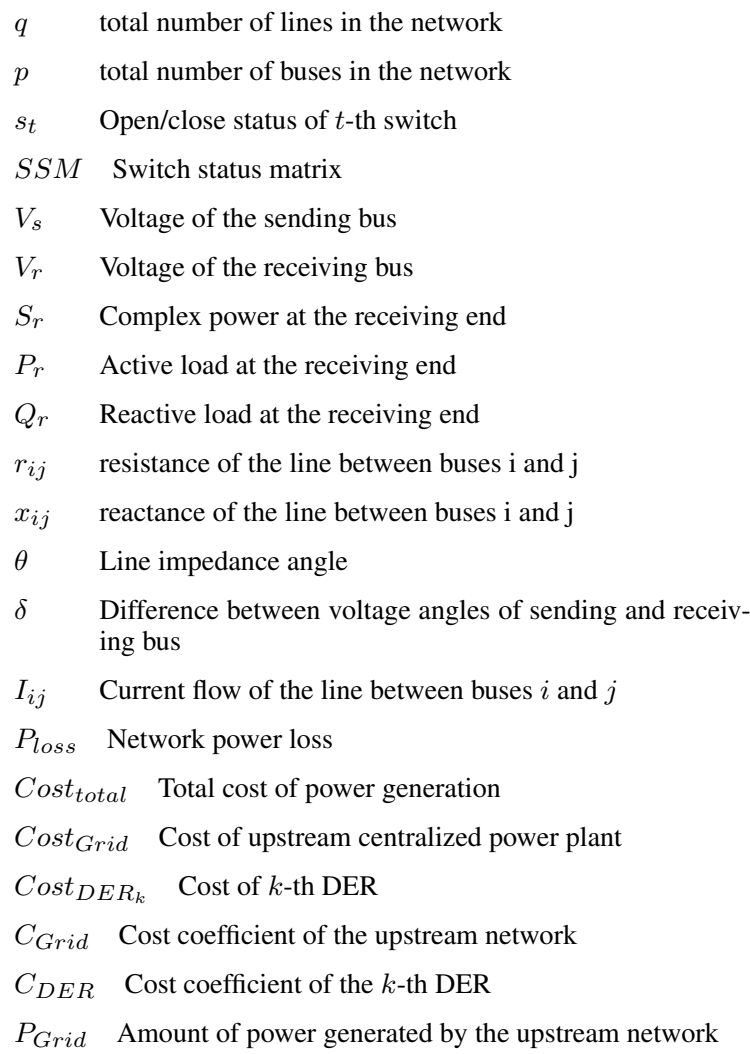




\section{$P_{D E R} \quad$ Amount of power generated by the $k$-th DER}

\section{Introduction}

\subsection{Aims and Scope}

High quality services to the end-users and continuity of electric power supply are the major targets in power network management. In this regard, assessing network reliability plays a pivotal role [1] such that the power delivery to the end-users must be continuously assessed, and decisions and preventive actions must be taken to increase the reliability indices of the network. Reliability assessment has commonly two major aspects of adequacy and security [2]. Adequacy, i.e. fulfilling the load demand of the end-users in all times, is concerned with static operating condition, rather than power systems dynamics and transient disturbances [2]. Widely-used indices for the adequacy including system average interruption duration index (SAIDI) and system average interruption frequency index (SAIFI), are used to show the network ability in providing necessary facilities to generate adequate energy with good delivery to the actual end-users' load point $[2,3]$. On the other side, security deals with network responses to all kinds of disturbances so is concerned with the network dynamics and transient operating conditions such as voltage instability in the event of major generation or/and transmission outage [2]. The ever expansion of smart grid makes the reliability assessment more complex and challenging. Increasing distributed energy resources (DERs) improve the power supply adequacy due to their inexhaustible nature, but it can reduce the security as a result of power injection and power flow increase of the lines in the distribution network [4]. To achieve an acceptable and optimal reliability, the old-fashion "fit and forget" strategy can no longer be applied in the modern smart networks. The DERs output powers must be frequently changed in different time horizons and operating conditions, where the network must be continuously reconfigured, i.e. network reconfiguration, to address such changes when needed [5].

\subsection{Literature Survey}

Network reconfiguration is represented as the action of changing topological structure of feeders by intelligently changing the status of tie and sectionalizing switches from open to close and vice versa, without altering the radial structure of the network. The reconfiguration must be done for a number of reasons including loss reduction $[6,7]$, voltage profile improvement $[8,9]$, reliability enhancement $[10,11]$, etc. With active DERs in the network, reconfiguration will become more complex especially if security is a concern.

A great deal of work in the literature focuses on enhancing the network reliability through network reconfiguration. In [12], for reliability enhancement, a reconfiguration technique is proposed using a novel neighborhood search algorithm. In [13], to improve reliability and reduce system losses, reconfiguration problem has been modeled in a binary structure where a binary particle swarm optimization is used to solve the problem. The reliability index used in this reference is energy not supplied (ENS). In [14] to increase the reliability and reduce the losses, the number of open lines has been considered equal to the number of tie switches to prove the radiality of the network. The paper only uses ENS as the reliability index in the formulations. In [15], Genetic Algorithm (GA) is used to optimize the reconfiguration problem where the reliability indices SAIFI and ENS are used in the problem formulations.

In [16], by considering network reconfiguration in the expansion planning problem an aggregated model is proposed and particle swarm optimization is used to solve the problem. In [17], enhanced GA is used to optimize a function with single loop formation strategy that provides only radial topologies. In their proposed approach, reliability and power loss are improved where the indices SAIDI and ENS are used to validate the problem formulation. In [18-22], power loss and reliability indices such as SAIFI, SAIDI, and ENS are considered as the power quality indicators and improved through network reconfiguration procedure. In [23], the problem of distribution network reconfiguration is modeled using a mixed integer linear programming approach to reduce power losses and improve network reliability.

In [24], for the network reconfiguration, a binary firefly algorithm is implemented to find the location of switches needed to be open. SAIFI, and momentary average interruption duration index (MAIDI) indices are used for the reliability evaluation. A further study is also proposed to investigate the effect of DER presence in the problem. In [25], by considering DERs an objective function is presented to enhance the reliability by minimizing the expected customer interruption costs and the power losses. To solve the presented multiobjective problem, modified artificial bee colony (ABC) optimization technique is used. Similarly, in [26-28], new frameworks for network reconfiguration of distribution network with integrated DERs are proposed to reduce power losses and improve reliability indices. In Table 1, a taxonomy of these studies are demonstrated.

\subsection{Contributions}

In all the studies mentioned in the previous section, only adequacy aspect of reliability is considered in the formulations. However, presence of DERs (and resulting power injection) and power flow increase of the lines in the distribution network can reduce security. Moreover, to establish a reliable and secure active network where DERs are generating power, their output power must be optimally determined. Therefore, in this paper, to consider the distribution network adequacy and security (DNAS) enhancement and loss reduction, DERs sizing are carried out simultaneously with the network reconfiguration. Basic concepts of graph theory is adopted for the suggested reconfiguration method. To address security concerns of DERs in the distribution network, a combined line and bus security index is then utilized. The indices SAIFI, SAIDI, and ENS are used to assess the adequacy aspect. To solve the overall objective function, a powerful new optimization tool, human behavior-based optimization (HBBO) [29], is employed. The main contributions of this paper can be summarized as follows:

- Reconfiguration and DERs sizing are carried out in tandem via an optimization problem to improve DNAS, a reliability enhancement from both aspects of adequacy and security,

- Graph theory is used for the design of the suggested reconfiguration method.

- A combined bus and line security index is used to overcome security concerns of DERs existence in the distribution network,

- A new optimization technique, $\mathrm{HBBO}$, is used for solving the optimization problem.

The rest of the paper is structured as follows: the problem formulation consists of reconfiguration method, reliability indices of adequacy and security, DERs presence consideration and overall objective function will be described in Section 2. In Section 3, HBBO will be described and in Section 4, test results and discussions will be given. Finally, in Section 5, the paper will be concluded.

\section{Problem formulation}

The aim of this section is to describe the problem formulation and the objective function. The objective function will be eventually used in the optimization problem.

\subsection{Reconfiguration Method}

To perform reconfiguration, the status of sectionalizing and tie switches must be intelligently changed. The most important criteria to consider is maintaining the radial structure of the distribution network. In some researches, reconfiguration has been performed in a binary model and optimization methods opt the best structure through $2^{n}$ network configuration in a system with $n$ switches $[30,31]$. This method costs a great deal of time burden and likely 
Table 1 Researches conducted on reliability improvement of distribution networks through reconfiguration

\begin{tabular}{|c|c|c|c|c|}
\hline \multirow[b]{2}{*}{ Ref. } & \multirow{2}{*}{$\begin{array}{c}\text { DER } \\
\text { Consideration }\end{array}$} & \multicolumn{2}{|c|}{ Reliability Aspects } & \multirow{2}{*}{$\begin{array}{c}\text { Test } \\
\text { Systems }\end{array}$} \\
\hline & & Adequacy & Security & \\
\hline [10] & NO & SAIFI-SAIDI-AENS & NO & 33-bus \\
\hline$[11]$ & NO & SAIFI-SAIDI-AENS & NO & 69 -bus \\
\hline [12] & NO & SAIFI-SAIDI-AENS & NO & 33-bus \\
\hline [13] & NO & ENS & NO & 33-bus, 123-bus \\
\hline$[14]$ & NO & ENS & NO & 69-bus \\
\hline$[15]$ & NO & ENS & NO & 33-bus, 69-bus \\
\hline [17] & NO & EENS-SAIFI-SAIDI & NO & 33-bus, 69-bus \\
\hline [18] & NO & SAIFI-SAIDI-ENS & NO & 17-bus, 33-bus \\
\hline [19] & NO & SAIFI-SAIDI-ENS & NO & 43-bus, 136 -bus \\
\hline [20] & NO & SAIFI-SAIDI-EENS & NO & 33-bus, 69-bus \\
\hline [21] & NO & ENS & NO & 33-bus, 70-bus \\
\hline [22] & NO & SAIFI-SAIDI-ENS & NO & 33-bus, 69-bus \\
\hline [23] & NO & SAIFI-SAIDI-AENS & NO & 33-bus, 83-bus \\
\hline [24] & YES & SAIFI-MAIFI & NO & 47-bus \\
\hline [25] & YES & SAIFI-SAIDI & NO & 69-bus \\
\hline [26] & YES & SAIFI-EENS & NO & 44-bus \\
\hline [27] & YES & SAIFI-AENS & NO & 32-bus \\
\hline [28] & YES & SAIFI-AENS & NO & 33-bus, 69-bus \\
\hline This Paper & YES & SAIFI-SAIDI-AENS & YES & 33-bus, 83-bus \\
\hline
\end{tabular}

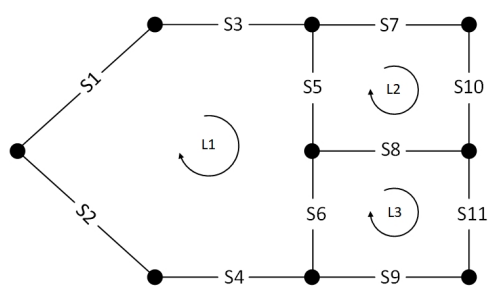

Fig. 1: Example of a simple network for analyzing the radial structure.

to trap in the local optimal solution. In the reconfiguration problem suggested here, graph theory is used to solve the said problems. Let each network configuration be a graph in which the network lines are the edges and the buses are the nodes of this graph. Since all the network configurations obtained from reconfiguration procedure must maintain its radial structure, and also all the buses must be supplied, it is concluded that (using the definition of tree in graph theory [32]), the graph of the reconfigured network must be a tree. Therefore, the following conditions must be met in each network structure post reconfiguration: 1 . If in a network, there was $p$ buses and $q$ lines, the following condition must be met:

$$
q=p-1
$$

2. No loop should exist in the network configuration. If open/close status of the switches and consequently the status of all lines be known and available, the status matrix of switches is defined as:

$$
S S M=\left[s_{1}, s_{2}, \ldots, s_{n}\right]
$$

For open switches $s=0$ and for close switches $s=1$. With the same approach, all system loops can be defined. For example, consider network configuration of Fig. 1, status matrix of switches in each loop (without basic loop) is described as:

$$
\begin{gathered}
L_{1}=\left[s_{1}, s_{3}, s_{5}, s_{6}, s_{4}, s_{2}\right] \\
L_{2}=\left[s_{5}, s_{7}, s_{10}, s_{8}\right] \\
L_{3}=\left[s_{6}, s_{8}, s_{11}, s_{9}\right] \\
L_{1,2}=\left(L_{1} \cup L_{2}\right)-\left(L_{1} \cap L_{2}\right) \\
L_{1,3}=\left(L_{1} \cup L_{3}\right)-\left(L_{1} \cap L_{3}\right)
\end{gathered}
$$

$$
L_{2,3}=\left(L_{2} \cup L_{3}\right)-\left(L_{2} \cap L_{3}\right)
$$

Now for satisfying the conditions in (1) and (2), in each loop one switch must be open and no common open switches between two or more loops must exist. Therefore, the system in Fig. 1 is radial when the following condition is met:

$$
\operatorname{sum}\left\{\operatorname{prod}\left(L_{1}\right), \operatorname{prod}\left(L_{2}\right) \operatorname{prod}\left(L_{3}\right), \ldots\right\}=0
$$

The above method for proving the radiality of the network is applicable in all networks, which cal also provide simplicity and less computational burden for the reconfiguration algorithm. The optimization algorithm will select only one switch from each loop and if the condition in (9) met, it considers this network configuration feasible and continues the process. The algorithm selects new set of open switches from each loop and continues the process until it reaches the global optima.

\subsection{Reliability Assessment}

As previously mentioned, adequacy deals with satisfying load demand of end-users in all times and is concerned with static operating condition where security deals with the network responses to all disturbances and is concerned with the dynamics or transient operating condition of the system, e.g. major generation and/or transmission outage causing voltage instability. In Section 1, we pointed out that the previous work in the reliability enhancement of the network using reconfiguration [10-17, 24], only focus on the adequacy aspect of reliability. Here we consider both aspects of DNAS to achieve a comprehensive reliability improvement.

2.2.1 Adequacy: Adequacy is commonly evaluated with four most popular indices namely SAIFI, SAIDI, CAIDI, and AENS. Their definitions are summarized below [33].

SAIFI represents the amount of interruption that an average enduser faces during a specific time. SAIFI is formulated as [2]:

$$
S A I F I=\frac{\text { number of end-user interruptions }}{\text { total number of end-user served }}
$$

SAIDI represents the time duration of the interruptions that the end-user faces. SAIDI is formulated as [2]:

$$
S A I D I=\frac{\text { sum of end-user interruption durations }}{\text { total number of end-users }}
$$

Note that the above adequacy indices do not provide any information about the not supplied energy of the end-users during an incident and only concentrate on the interruptions experienced by them. Therefore, in the reliability assessment of the problem formulation, AENS index is modeled as [2]:

$$
A E N S=\frac{\text { energy not served during interruptions }}{\text { total number of end-user served }}
$$

To improve adequacy, the indices must be minimized. In this paper as DERs exists, both SAIFI and AENS indices are minimized in the optimization procedure by minimizing the following adequacy index of reliability $(\mathrm{AI})$ :

$$
A I=c_{1} \times S A I F I+c_{2} \times A E N S
$$

where, $c_{1}$ and $c_{2}$ are the importance factors and valued at 0.5 in this study.

2.2.2 Security: Voltage security (especially voltage stability) is the most important factor of the network security that can limit available power sending capability [34]. To include voltage stability in the problem formulation, a combined index for both bus and line stability is used. The combined index is written just after the expression of each of bus voltage stability index (BSI) and line stability index (LSI). For bus voltage stability, the approach presented 


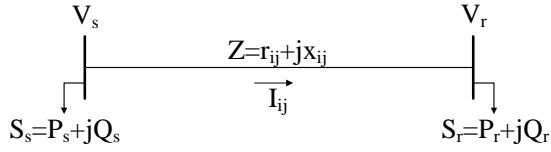

Fig. 2: Electric equivalent of a radial distribution feeder.

in [35] is used to identify the weakest voltage of buses in the network. Consider the electric equivalent of a radial distribution feeder, depicted in Fig. 2. In this equivalent network, based on the load flow technique presented in [36] for radial distribution networks, the following equations can be written:

$$
\begin{gathered}
I_{i j}=\frac{V_{s}-V_{r}}{r_{i j}+j x_{i j}} \\
I_{i j}=\frac{P_{r}-j Q_{r}}{V_{r}^{*}}
\end{gathered}
$$

where, $I_{i j}$ is the current flowing through the line between buses i and j. Also, $V_{s}$ and $V_{r}$ are the sending and receiving bus voltages, respectively. Moreover, $P_{r}$ and $Q_{r}$ are active and reactive loads at receiving end, and also $r_{i j}$ and $x_{i j}$ are the resistance and reactance of the line between buses i and j. Using (14) and (15), after some calculations (see Appendix), the following equation is obtained:

$$
\left|V_{r}\right|^{4}-B\left|V_{r}\right|^{2}+C=0
$$

where,

$$
\begin{gathered}
B=\left|V_{s}\right|^{2}-2\left(P_{r} r_{i j}-Q_{r} x_{i j}\right) \\
C=\left(P_{r}^{2}+Q_{r}^{2}\right)\left(r_{i j}^{2}+x_{i j}^{2}\right)
\end{gathered}
$$

From (16), it can be concluded that for a feasible load flow solution in the radial distribution network the following criteria must be met:

$$
B^{2}-4 C \geq 0
$$

Based on the above equation, BSI for $i$-th bus is written as follows:

$$
\begin{array}{r}
B S I_{i}=\left|V_{s}\right|^{4}-4 \times\left(P_{r} x_{i j}-Q_{r} r_{i j}\right)^{2}- \\
4 \times\left|V_{s}\right|^{2} \times\left(P_{r} r_{i j}+Q_{r} x_{i j}\right)^{2}
\end{array}
$$

The best condition for the stable network operation achieves when the BSI values become closer to one. The value of less than zero for BSI, means instability in network operating condition. In the proposed security improvement method, the value of BSI is calculated for each bus and, the bus with the lowest BSI value will be then put in the first part of the final voltage stability index.

For LSI, the approach presented in [37] is used to identify the weakest line in the network which is more likely to threat system stability when load increases. In this way, power flow equations for $\pi$ model representation of the line is utilized and the following equation for complex power at receiving end is obtained:

$$
S_{r}=\frac{\left|V_{s}\right|\left|V_{r}\right|}{|Z|} \angle(\theta-\delta)-\frac{V_{r}^{2}}{|Z|} \angle \theta
$$

where, $|Z|$ and $\theta$ are the line impedance amplitude and angle respectively. $S_{r}$ is the complex power of receiving end. $\delta$ is the difference between sending and receiving bus voltage angles. By separating the real and reactive powers from (21), the following equations can be written:

$$
P_{r}=\frac{\left|V_{s}\right|\left|V_{r}\right|}{|Z|} \cos (\theta-\delta)-\frac{V_{r}^{2}}{|Z|} \cos \theta
$$

$$
Q_{r}=\frac{\left|V_{s}\right|\left|V_{r}\right|}{|Z|} \sin (\theta-\delta)-\frac{V_{r}^{2}}{|Z|} \sin \theta
$$

using (23), the voltage at the receiving end can be calculated as follows:

$$
V_{r}=\frac{V_{s} \sin (\theta-\delta) \pm \sqrt{\left[V_{s} \sin (\theta-\delta)\right]^{2}-4 Q_{r} x_{i j}}}{2 \sin \theta}
$$

To have real values for $V_{r}$ in (24), the following criteria must be met:

$$
\left[V_{s} \sin (\theta-\delta)\right]^{2} \geq 4 Q_{r} x_{i j}
$$

or:

$$
\frac{4 Q_{r} x_{i j}}{\left[V_{s} \sin (\theta-\delta)\right]^{2}} \leq 1
$$

Based on (26), LSI for the line between buses $i$ and $j$ can be written as follows:

$$
L S I_{i j}=\left(4 \times x_{i j} \times Q_{r}\right) /\left(V_{s} \sin (\theta-\delta)\right)^{2}
$$

LSI models the effect of line stability on voltage stability. Based on the mentioned BSI and LSI, voltage collapse can be accurately predicted. Until LSI value becomes lower than one, the system is stable. Values of one or higher for the LSI means an unstable network. It is evident that BSI and LSI are the opposite of each other i.e. upper value for BSI is more desirable while the lower value for LSI is better. In this paper, these two indices are combined and overall voltage stability index (OVSI) is obtained as follows:

$$
O V S I=c_{3} \times \max \{L S I\}+c_{4} / \min \{B S I\}
$$

where, $c_{3}$ and $c_{4}$ are the weighting factors and valued at 0.5 in this study. In order to improve the security aspect of reliability, OVSI must be minimized.

\subsection{Network Power Loss}

Network power loss for each configuration is typically calculated by the equation [6]:

$$
P_{\text {loss }}=\sum r_{i j} \times I_{i j}^{2}
$$

In the problem, minimization of power loss is another objective. Forward-backward sweep load flow is used in this paper to perform load flow analysis needed in the problem formulation. Forwardbackward sweep load flow is suitable for load flow calculation in the distribution networks [38].

\subsection{Cost of Power Generation}

Minimizing the total cost of power generation or reducing the operation cost, is an important goal of the distribution system management. Let the cost of $k$-th DER be equal to $\operatorname{Cost}_{D E R_{k}}$ and the 
cost of upstream centralized power plants be equal to Cost $_{\text {Grid }}$, then the total power generation costs is calculated as:

$$
\operatorname{Cost}_{\text {total }}=\operatorname{Cost}_{\text {Grid }}+\sum_{k=1}^{N_{D E R}} \operatorname{Cost}_{D E R_{k}}
$$

where NDER is the number of DERs and:

$$
\begin{gathered}
\text { Cost }_{\text {Grid }}=C_{\text {Grid }} \times P_{\text {Grid }} \\
\text { Cost }_{D E R_{k}}=C_{D E R_{k}} \times P_{D E R_{k}}
\end{gathered}
$$

where $C_{G r i d}$ and $C_{D E R_{k}}$ are the cost coefficients of network and $k$-th DER respectively. $P_{G r i d}$ and $P_{D E R_{k}}$ are the amount of power taken from the upstream network and $k$-th DER, respectively.

\subsection{Overall Objective Function of the Problem}

Objective function of the problem is to find suitable status of switches and sizes of DERs that can improve network reliability and reduce power losses. According to the reconfiguration method discussed in Subsection 2.1, only one open switch in each loop must be optimally selected where the optimal sizes of DERs must be also found. As a result, the said problem becomes an optimization problem in which the decision variable matrix is:

$$
X=\left[O S_{1}, O S_{2}, \ldots, O S_{i}, P G_{1}, P G_{2}, \ldots, P G_{j}\right]
$$

where $O S_{i}$ is the selected switch in loop $i$ that should be opened to perform reconfiguration process and $P G_{j}$ is the size of $j$-th DER power generation. To improve system reliability, in Subsection II.B, DNAS aspects were discussed and their indices were presented. For improving network reliability OVSI value must be minimized and for reducing power loss and operation cost, values of $P_{l o s s}$ and Cost $_{\text {total }}$ must be minimized. As the values of OVSI, $P_{\text {loss }}$ and Cost $_{\text {total }}$ depend on the network configuration and DERs sizes, they can be expressed as a function of X, i.e. $O V S I(X), P_{\text {loss }}(X)$, and $\operatorname{Cost}_{\text {total }}(X)$. The overall objective function of the problem is then described as:

$$
\begin{array}{r}
O F=\left(w_{1} \times A I(X)\right)+\left(w_{2} \times O V S I(X)\right)+ \\
\left(w_{3} \times P_{\text {loss }}(X)\right)+\left(w_{4} \times \text { Cost }_{\text {total }}(X)\right)
\end{array}
$$

where $w_{1}, w_{2}, w_{3}$, and $w_{4}$ are the weighting factors of the objective functions. Minimizing the overall objective function is an optimization problem. In this paper, a new powerful optimization algorithm, human behavior-based optimization (HBBO) [29] method is used which will be described in the subsequent section.

\section{Human behavior-based optimization}

HBBO is a novel meta-heuristic optimization method aiming at artificially simulating the behavior of a human in the pursuit of his goals [29]. Meta-heuristic algorithms are a kind of random algorithm used to find optimal value of a function. Optimization methods and algorithms are categorized into two groups of exact algorithms and approximate algorithms. Exact algorithms are capable of finding the global optimal solution, but they are not efficient enough for difficult optimization problems and their execution time expands exponentially with the dimensions of the problems.

Approximate algorithms are capable of finding good (near optimal) solutions at short time for complex optimization problems. Approximate algorithms are also classified into two categories: heuristic algorithms, and meta-heuristic algorithms. The two main problems of heuristic algorithms, poor performance for escaping from local optima, and fast converging to these points. Metaheuristic algorithms are presented to solve these problems. In fact, meta-heuristic algorithms are one of a kind of approximate optimization algorithms that have solutions to escape from the local optima and can be applied to a wide range of issues. Various types of this algorithm have been developed in the last decades, and HBBO seems suitable for power system problems which has been recently introduced.

Learning via education, correcting behavior via consulting and also the probability of changing the way of thinking are some major human behaviors which HBBO implements [29]. This optimization method consists of five steps:

\section{Step 1 - Initialization:}

In this step, initial individuals that are problem decision variables matrix are created and randomly spread among the fields. For our problem, an individual is just the matrix of decision variables (X).

$$
\text { Individual }=\left[O S_{1}, O S_{2}, \ldots, O S_{i}, P G_{1}, P G_{2}, \ldots, P G_{j}\right]
$$

\section{Step 2 - Education:}

Simulation of improvement through education is the goal of this step. In this way, the best individual of each field, or an expert individual, is found and other individuals of the field will try to learn from them by moving toward them. This movement is not a direct move, and the individual finds a place around the expert from $r_{\text {min }}$ to $r_{\max }$. To perform this step, a spherical coordinate system for $\mathrm{N}$ dimensional Euclidean space is implemented.

\section{Step 3 - Consultation:}

Finding random advisors by all the individuals and consulting with them is the core to the consultation. To briefly explain the consultation procedure, an advisor randomly selects some decision variables from the individual variable matrix and change them to what he thinks is better. In advanced mode of HBBO, after changing variables, the objective function of this new set of variables will be calculated and in case of achieving better value the consultation will be effective and the variables will be updated.

\section{Step 4 - Field Changing Probability:}

In this step, using a rank probability method, in some fields an individual's field may be changed to model the changes that people may experience during their lives.

\section{Step 5 - Finalization:}

As the variables matrix of the individuals changes during each iteration of the algorithm, in this step, individuals' fitness function will be updated. In addition, if the stopping conditions met, the algorithm will be stopped.

The reconfiguration model proposed in this paper is a nonlinear formulation and therefore a meta-heuristic optimization technique is selected to solve it. This kind of optimization techniques do not guarantee to obtain the global optima and may trapped in a local one, but having the advantage of formulating the complete detail in a nonlinear model. It is also possible to linearize the model and neglect some features in it, and then solve it with a mathematical approach to guarantee reaching the global optima. In this way, some simplifications are made in the formulation which decrease the accuracy of the obtained result. There is always a controversy in choosing between these two approaches but for the large models where linearization causes the significant error, using meta-heuristic approaches is a better choice. For this reason, meta-heuristic approach is selected here to conduct the optimization task.

\section{Simulation results and discussion}

Comprehensive test studies are carried out in this section to validate the proposed approach using two practical networks, i.e. the IEEE 33-bus network and 83-bus network of TPC. 
The process of solving an optimization problem has three stages, each of which has different steps. In each step, some activities must be done to complete that step. First step is a preparation in which we need to get a clear understanding of the problem of interest, and set the design objectives of the optimization algorithm. The next step is the selection strategy, and the definition of performance measurement criteria. In this step, the problem objective function must be defined. The final stage is the implementation of the optimization algorithm according to the designed objective function in the previous step.

The procedure of $\mathrm{HHBO}$ algorithm implementation for the reconfiguration problem in this study is described as follows:

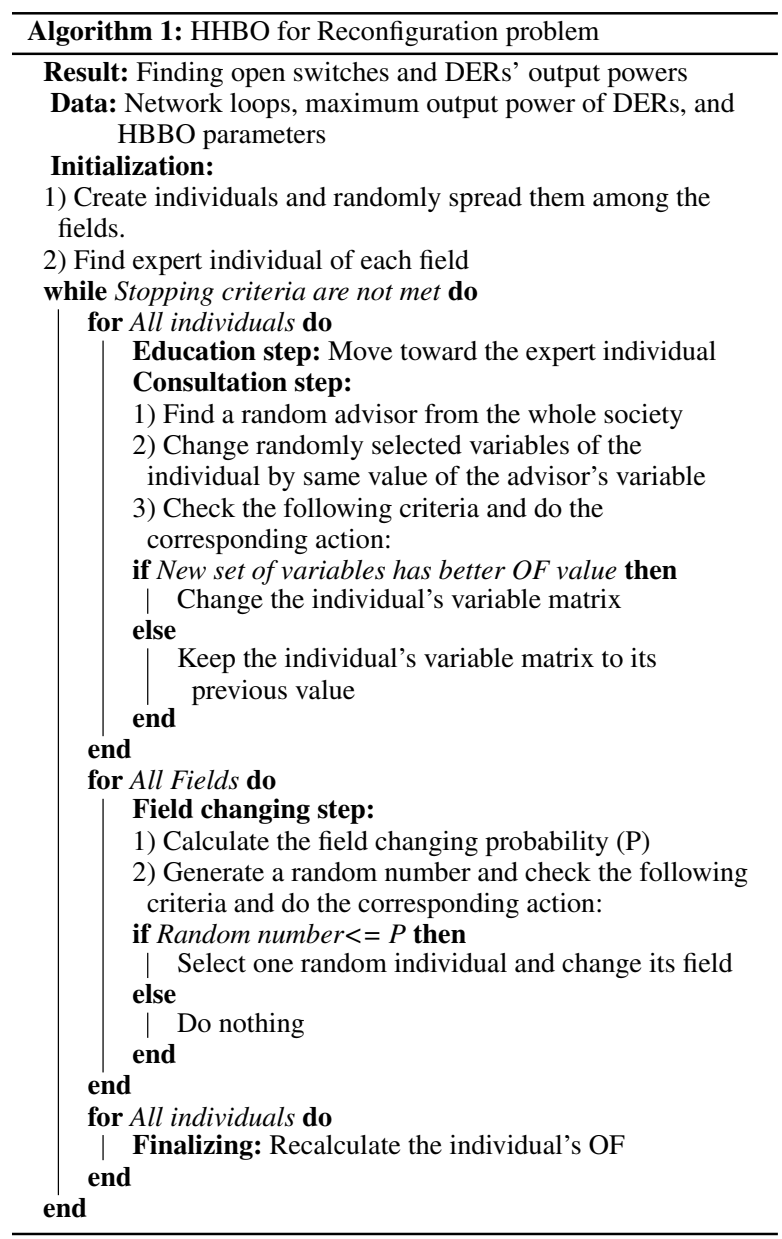

To show the advantage of using HBBO algorithm in our study, a comparison with GA is carried out. For GA, Matlab optimization toolbox with adaptive feasible mutation and scattered crossover is used. The population and generations size are set to 150 , and 30 respectively. Other control parameters of GA are set to be the same as default values of the toolbox. Parameters of $\mathrm{HBBO}$ are given in Table 2 .

Table 2 HBBO parameters

\begin{tabular}{lc}
\hline Parameter & Value \\
\hline Population size & 150 \\
Field size & 30 \\
$k_{1}$ & 0 \\
$k_{2}$ & 2.5 \\
$\sigma$ & 0.2 \\
Optimization mode & advance \\
\hline
\end{tabular}

\subsection{IEEE 33-bus radial distribution network}

The IEEE 33-bus network with $12.66 \mathrm{kV}$ voltage level [39] is selected to conduct the first part of test studies. The line and load data of the candidate test network is found in [39]. It is assumed that all lines have sectionalizing switches, so there are 32 sectionalizing switches, and 5 tie switches in the network. This network with the location of its DERs is shown in Fig. 3. To test the proposed algorithm in different conditions, the three following scenarios are considered:

Scenario 1: Standard network without performing network reconfiguration.

Scenario 2: Performing the proposed reconfiguration approach for the test system without utilizing DERs.

Scenario 3: Similar to scenario 2, but with utilizing DERs in the network.

For reliability assessment, failure rates of the lines are calculated based on their impedance value. In this way, the line with the maximum impedance value has the maximum failure rate of 0.4 (failure/year) and the line with the minimum impedance value has the minimum failure rate of 0.1 (failure/year). For other lines, failure rates are calculated proportionally based on these minimum and maximum rates. Repair time for all lines is supposed to be 8 hours. Other network components such as DERs, transformers, circuit breakers, etc. are assumed to be fully reliable. End-user data are also given in Table 3.

Table 3 End-user data of IEEE 33-bus network

\begin{tabular}{cccc}
\hline $\begin{array}{c}\text { Bus } \\
\text { Number }\end{array}$ & $\begin{array}{c}\text { Number of } \\
\text { End-Users }\end{array}$ & $\begin{array}{c}\text { Bus } \\
\text { Number }\end{array}$ & $\begin{array}{c}\text { Number of } \\
\text { End-Users }\end{array}$ \\
\hline 1 & 450 & 17 & 350 \\
2 & 350 & 18 & 400 \\
3 & 550 & 19 & 300 \\
4 & 300 & 20 & 400 \\
5 & 300 & 21 & 500 \\
6 & 950 & 22 & 400 \\
7 & 950 & 23 & 1450 \\
8 & 500 & 24 & 1250 \\
9 & 500 & 25 & 250 \\
10 & 350 & 26 & 450 \\
11 & 250 & 27 & 250 \\
12 & 350 & 28 & 550 \\
13 & 500 & 29 & 850 \\
14 & 250 & 30 & 750 \\
15 & 400 & 31 & 1000 \\
16 & 250 & 32 & 250 \\
\hline
\end{tabular}

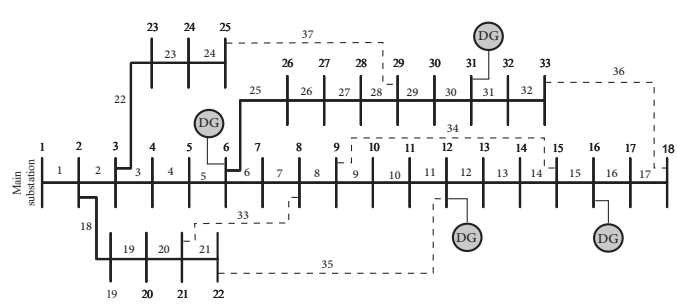

Fig. 3: IEEE 33-bus network with DERs locations.

To use the reconfiguration method expressed in Subsection 2.1, the network loops must be specified. At first, it is assumed that all switches are closed. After the optimization algorithm procedure starts, one switch from each loop is selected by considering the criteria discussed in Subsection 2.1, mainly by satisfying the criteria of equation (9) which guarantees the radiality of the network. As seen in Fig. 3, IEEE 33-bus system has five loops. Optimization algorithms are to find one independent switch from each loop in which the overall objective function (20) becomes minimum. By applying $\mathrm{HBBO}$ and GA, the results are obtained. Open switches number that represent the configuration of the network, reliability indices of 
Table 4 Results of the conducted study on IEEE 33-bus distribution network

\begin{tabular}{|c|c|c|c|c|c|}
\hline & \multirow[t]{2}{*}{ Scenario 1} & \multicolumn{2}{|l|}{ Scenario 2} & \multicolumn{2}{|l|}{ Scenario 3} \\
\hline & & HBBO & GA & HBBO & GA \\
\hline $\begin{array}{l}\text { Open switches } \\
\text { SAIFI (failure/year) } \\
\text { SAIDI (hour) } \\
\text { AENS } \\
\text { OVSI } \\
\text { LSI } \\
\text { BSI } \\
\text { Power loss (kW) } \\
\text { Voltage of the weakest bus }(\%) \\
\text { LCU of the weakest line }\end{array}$ & $\begin{array}{l}33-34-35-36-37 \\
1.2672 \\
10.1372 \\
1.9728 \\
0.6305 \\
0.0208 \\
0.8063 \\
202.67 \\
90.38 \\
1\end{array}$ & \begin{tabular}{l|}
$7-9-14-32-37$ \\
1.1404 \\
9.1229 \\
1.9690 \\
0.65515 \\
0.02118 \\
0.7757 \\
139.55 \\
93.78 \\
0.9827
\end{tabular} & $\begin{array}{l}7-11-14-32-37 \\
1.1449 \\
9.1588 \\
1.9737 \\
0.65515 \\
0.02118 \\
0.7757 \\
141.21 \\
93.78 \\
0.9827\end{array}$ & $\begin{array}{l}7-9-14-36-28 \\
1.0469 \\
8.3753 \\
1.8050 \\
0.61211 \\
0.02066 \\
0.83086 \\
86.87 \\
95.44 \\
0.7835\end{array}$ & $\begin{array}{l}7-9-14-32-28 \\
1.0605 \\
8.4839 \\
1.8311 \\
0.60905 \\
0.02065 \\
0.8351 \\
91.89 \\
95.52 \\
0.8225\end{array}$ \\
\hline
\end{tabular}

SAIFI, SAIDI, and AENS, and also OVSI, LSI, BSI, weakest bus voltage, and line capacity usage (LCU) ratio of the weakest line, which has the highest current, for the obtained configuration, to the normal operation configuration, and finally total network power loss are tabulated in Table 4 .

For scenarios 3, where DERs are generating power in the network, output power generation of DERs obtained from optimization algorithms and their maximum sizes are given in Table 5. The execution times of HBBO and GA algorithms for this scenario are 18.12 and 19.46 seconds. The evolutionary progresses of objective function for GA and HBBO are depicted in Fig. 4. For all scenarios, the voltage profiles of the selected network using HBBO method are shown in Fig. 5.

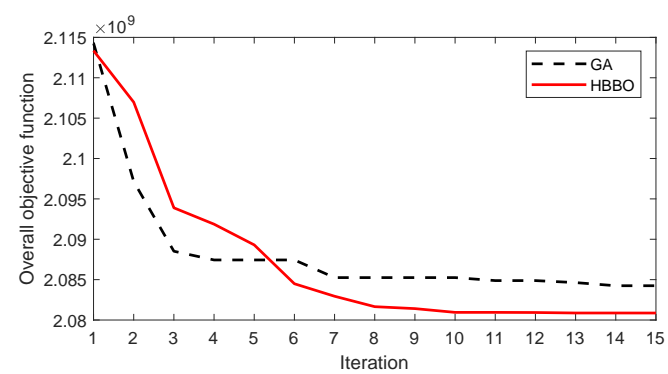

Fig. 4: Evolutionary progress of objective function values for GA and $\mathrm{HBBO}$.

Table 5 Maximum power generation and optimal sizes of DERs in 33-bus distribution network (KW)

\begin{tabular}{llll}
\hline & Maximum & \multicolumn{2}{c}{ Scenario 3 } \\
\cline { 3 - 4 } DER Locations & Possible Generation & HBBO & GA \\
\hline DER on bus 6 & 500 & 183.20 & 32.84 \\
DER on bus 12 & 300 & 211.55 & 101.18 \\
DER on bus 16 & 300 & 183.54 & 271.45 \\
DER on bus 31 & 500 & 479.26 & 417.98 \\
\hline
\end{tabular}

From data given in Table III, by comparing scenario 1 with scenario 2 , it can be seen that reconfiguration helps improving network reliability and reducing power losses. In addition, by considering the security aspect in reliability, minimum voltage of the network buses is increased without threatening security indices of OVSI, LSI, and BSI. This means more voltage security.

In scenario 3, the DERs are present actively and their generation sizes are optimally selected by HBBO and GA, a trade-off between security and power generation of DERs takes place. By looking at Tables 4,and 5, it is observed that the DERs are producing power and the power loss of the system reduces and voltage improves while the LCU ratio of weakest line using HBBO is 0.7835 and also security indices of OVSI, LSI, and BSI are in a good range even better than

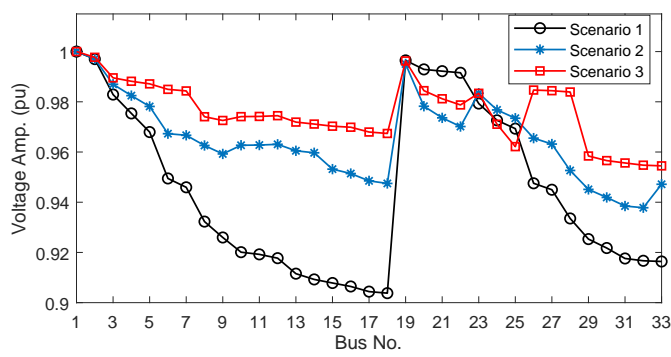

Fig. 5: Voltage profile of network in all scenarios.

the base case. The line with LSI value near one or BSI value near zero is considered as the critical line. This value for LCU ratio shows that all of the lines, even the weakest line which has the maximum current, is in their allowed range. This proves that by taking into account the security aspect in the problem formulations as a factor of reliability helps the network to benefit from the reconfiguration tool for using the capacity of DERs.

The minimum voltage of network (see Table 4) demonstrate that security consideration can also help voltage improvement. Looking at Tables 4 , and 5, with taking the security into account (in the formulations) helps to securely utilize DERs in the network. Comparison of HBBO and GA results in scenario 3, which is more complex than the other scenarios, indicates that HBBO algorithm outperforms GA in hard optimization problems and is especially suitable for our problem. Voltage profiles for all scenarios are shown in Fig. 5, and as seen, in scenario 3 , the best performance can be achieved where the voltage profile of the system improves significantly.

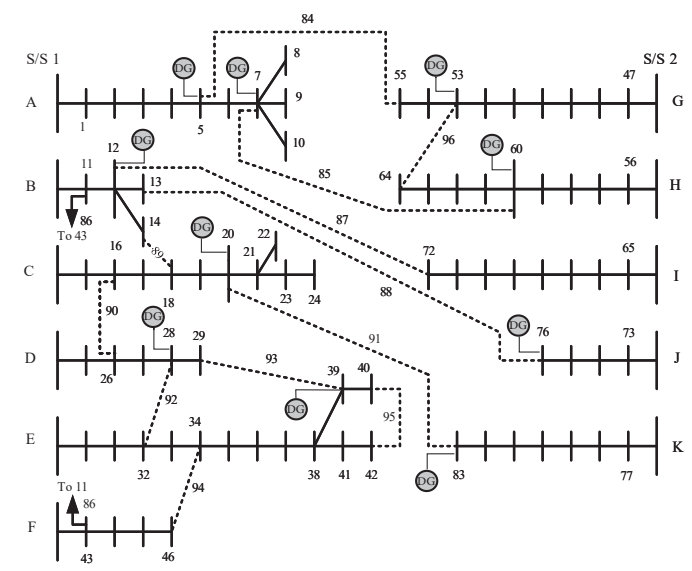

Fig. 6: 83-bus network of TPC with DERs locations. 


\subsection{3-bus distribution network of TPC}

To evaluate the performance of the proposed method for larger practical distribution networks, other studies are carried out on 83-bus distribution network of TPC, depicted in Fig. 6 [40]. This network is a $11.4 \mathrm{kV}$ system with 11 feeders. The line and load data could be found in [40]. Similar to the previous test network, it is assumed that all lines have sectionalizing switches, so there are 83 sectionalizing switches, and 13 tie switches in the network. Totally, 10 DERs on the buses $5,7,12,20,28,39,53,60,76$, and 83 with maximum power capacity of $500 \mathrm{~kW}$ are considered in this network. For failure rates of the lines, similar approach of the previous test network is used. End-user data are given in Table 6. Other parameters are same as the previous test network.

Table 6 End-user data of 83-bus network of TPC

\begin{tabular}{|c|c|c|c|c|c|}
\hline $\begin{array}{l}\text { Bus } \\
\text { Number }\end{array}$ & $\begin{array}{l}\text { Number of } \\
\text { End-Users }\end{array}$ & $\begin{array}{c}\text { Bus } \\
\text { Number }\end{array}$ & $\begin{array}{l}\text { Number of } \\
\text { End-Users }\end{array}$ & $\begin{array}{c}\text { Bus } \\
\text { Number }\end{array}$ & $\begin{array}{l}\text { Number of } \\
\text { End-Users }\end{array}$ \\
\hline 1 & 0 & 29 & 100 & 57 & 15 \\
\hline 2 & 50 & 30 & 0 & 58 & 200 \\
\hline 3 & 350 & 31 & 400 & 59 & 0 \\
\hline 4 & 300 & 32 & 100 & 60 & 10 \\
\hline 5 & 400 & 33 & 100 & 61 & 10 \\
\hline 6 & 800 & 34 & 500 & 62 & 100 \\
\hline 7 & 600 & 35 & 150 & 63 & 250 \\
\hline 8 & 450 & 36 & 200 & 64 & 250 \\
\hline 9 & 450 & 37 & 50 & 65 & 0 \\
\hline 10 & 450 & 38 & 50 & 66 & 25 \\
\hline 11 & 0 & 39 & 50 & 67 & 0 \\
\hline 12 & 300 & 40 & 50 & 68 & 200 \\
\hline 13 & 350 & 41 & 250 & 69 & 0 \\
\hline 14 & 350 & 42 & 200 & 70 & 0 \\
\hline 15 & 0 & 43 & 0 & 71 & 700 \\
\hline 16 & 150 & 44 & 15 & 72 & 200 \\
\hline 17 & 250 & 45 & 300 & 73 & 0 \\
\hline 18 & 350 & 46 & 100 & 74 & 0 \\
\hline 19 & 600 & 47 & 0 & 75 & 200 \\
\hline 20 & 450 & 48 & 0 & 76 & 100 \\
\hline 21 & 300 & 49 & 0 & 77 & 0 \\
\hline 22 & 100 & 50 & 50 & 78 & 100 \\
\hline 23 & 100 & 51 & 100 & 79 & 100 \\
\hline 24 & 100 & 52 & 250 & 80 & 50 \\
\hline 25 & 25 & 53 & 250 & 81 & 250 \\
\hline 26 & 25 & 54 & 250 & 82 & 50 \\
\hline 27 & 25 & 55 & 200 & 83 & 500 \\
\hline 28 & 700 & 56 & 0 & & \\
\hline
\end{tabular}

Results of applying $\mathrm{HBBO}$ to the reconfiguration problem of 83bus TPC network, are given in Tables 7 and 8 . Similar to the previous studies, by comparing scenarios 1 and 2 , we can see by using the proposed framework which includes the security aspect in the reliability formulations, not only the security indices i.e. OVSI, LSI, and BSI are not violated but also the minimum voltage of the network is improved. Besides, in scenario 3 where the DERs are actively generate power in the network, by simultaneously performing network reconfiguration and DERs sizing, the best condition is achieved where the power loss is minimized, voltage of the weakest bus and network reliability from both aspects of adequacy and security is improved. Also we can see the conditions for securely utilizing DERs in the network becomes provided by considering security aspect in the formulation of reliability improvement (see Tables 7 and 8). All in all, the conducted studies on 83-bus network of TPC, prove the applicability of the proposed method for large-scale distribution networks.

Normally in the power system operation and planning studies, there are some parameters that are based on the forecast values[41] and therefore are uncertain. The customers and substation loads are a sample of these uncertain parameters. Although, in this work it is supposed that the load values are fixed, but to tackle the load uncertainties, the worst-case scenario for the load demand is considered. The results obtained for the network configuration becomes more secure but lower optimal. Therefore, in our future work load and other influencing uncertainties will be considered by a proper stochastic approach[42-44].
Table 7 Results of the conducted study on 83-bus network of TPC

\begin{tabular}{llll}
\hline & Scenario 1 & Scenario 2 & Scenario 3 \\
\hline Open switches & $84-85-86-$ & $7-13-33-$ & $7-13-14-$ \\
& $87-88-89-$ & $37-42-62-$ & $34-38-40-$ \\
& $90-91-92-$ & $82-84-86-$ & $63-71-83-$ \\
& $93-94-95-$ & $87-89-90-$ & $84-86-90-$ \\
SAIFI (failure/year) & 96 & 92 & 92 \\
SAIDI (hour) & 1.1588 & 0.9798 & 0.82457 \\
AENS & 9.2707 & 7.8385 & 6.5966 \\
OVSI & 12.4824 & 12.7322 & 10.0162 \\
LSI & 0.67731 & 0.62531 & 0.60497 \\
BSI & 0.017974 & 0.017297 & 0.017374 \\
Power loss (kW) & 0.74814 & 0.81081 & 0.83853 \\
Voltage of the weakest bus (\%) & 531.994 & 478.332 & 416.5861 \\
LCU of the weakest line & 92.85 & 94.88 & 95.63 \\
& 1 & 0.9043 & 0.8653 \\
\hline
\end{tabular}

Table 8 Optimal sizes of DERs in 83-bus network of TPC (KW)

\begin{tabular}{llll}
\hline DER Location & Power Generation & DER Location & Power Generation \\
\hline DER on bus 5 & 315.35 & DER on bus 39 & 482.76 \\
DER on bus 7 & 74.67 & DER on bus 53 & 500 \\
DER on bus 12 & 492.03 & DER on bus 60 & 500 \\
DER on bus 20 & 307.66 & DER on bus 76 & 489.39 \\
DER on bus 28 & 495.25 & DER on bus 83 & 498.31 \\
\hline
\end{tabular}

\section{Conclusions}

In a distribution network with DERs present, a comprehensive reliability assessment must be carried out on the two aspects of adequacy and security. However, achieving the best reliability is difficult since the presence of DERs can improve adequacy but it may also jeopardize the security of the network. We propose a simultaneous reconfiguration and DERs sizing framework to improve both DNAS aspects of the reliability problem. The suggested reconfiguration routine is based on graph theory. To tackle security concerns of DERs existence in the network, a combined bus and line security index is further used. Problem is formulated in an objective function and the powerful optimization method, i.e. HBBO, is used to optimize the presented objective function. Obtained results validates our claims that the reliability and power losses is largely improved using the proposed approach. In our future works, we will demonstrate the feasibility of applying the proposed method for distribution systems with secondary networks, and with different sources of uncertainties and unbalancing condition.

\section{Acknowledgment}

This work was supported in part by the InteGRIDy project. The inteGRIDy project has received funding from the European Union's H2020 research and innovation programme under the grant agreement No. 731268.

\section{References}

1 H. Arasteh, V. Vahidinasab, M. S. Sepasian, and J. Aghaei, "Stochastic system of systems architecture for adaptive expansion of smart distribution grids," IEEE Transactions on Industrial Informatics, vol. 15, no. 1, pp. 377-389, 2019.

2 R. N. Allan et al., Reliability evaluation of power systems. Springer Science \& Business Media, 2013.

3 S. M. Hashemi, V. Vahidinasab, M. S. Ghazizadeh, and J. Aghaei, "Valuing consumer participation in security enhancement of microgrids," IET Generation, Transmission \& Distribution, vol. 13, no. 5, pp. 595-602, 2018.

4 A. Heidari, V. G. Agelidis, M. Kia, J. Pou, J. Aghaei, M. Shafie-Khah, and J. P. S. Catalão, "Reliability optimization of automated distribution networks with probability customer interruption cost model in the presence of dg units," IEEE probability customer interruption cost model in the presence of dg
Transactions on Smart Grid, vol. 8, no. 1, pp. 305-315, Jan 2017.

5 T. Niknam, A. Kavousifard, and J. Aghaei, "Scenario-based multiobjective distribution feeder reconfiguration considering wind power using adaptive modified particle swarm optimisation," IET Renewable Power Generation, vol. 6, no. 4, pp. 236-247, 2012.

6 R. S. Rao, K. Ravindra, K. Satish, and S. V. L. Narasimham, "Power loss minimization in distribution system using network reconfiguration in the presence of distributed generation," IEEE Transactions on Power Systems, vol. 28, no. 1, pp. 317-325, Feb 2013. 
7 F. V. Gomes, S. Carneiro, J. L. R. Pereira, M. P. Vinagre, P. A. N. Garcia, and L. R. De Araujo, "A new distribution system reconfiguration approach using optimum power flow and sensitivity analysis for loss reduction," IEEE Transactions on Power Systems, vol. 21, no. 4, pp. 1616-1623, Nov 2006.

8 Y.-T. Hsiao and C.-Y. Chien, "Multiobjective optimal feeder reconfiguration," IEE Proceedings-Generation, Transmission and Distribution, vol. 148, no. 4, pp. $333-$ 336,2001

9 Y. Liu, J. Li, and L. Wu, "Coordinated optimal network reconfiguration and voltage regulator/der control for unbalanced distribution systems," IEEE Transactions on Smart Grid, pp. 1-1, 2018.

10 A. Kavousi-Fard, T. Niknam, M.-R. Akbari-Zadeh, and B. Dehghan, "Stochastic framework for reliability enhancement using optimal feeder reconfiguration," Journal of Systems Engineering and Electronics, vol. 25, no. 5, pp. 901-910, 2014.

11 A. Kavousi-Fard and T. Niknam, "Optimal distribution feeder reconfiguration for reliability improvement considering uncertainty," IEEE Transactions on Power Delivery, vol. 29, no. 3, pp. 1344-1353, 2014

12 P. Zhang, W. Li, and S. Wang, "Reliability-oriented distribution network reconfiguration considering uncertainties of data by interval analysis," International Journal of Electrical Power \& Energy Systems, vol. 34, no. 1, pp. 138-144, 2012

13 B. Amanulla, S. Chakrabarti, and S. Singh, "Reconfiguration of power distribution systems considering reliability and power loss," IEEE transactions on power delivery, vol. 27, no. 2, pp. 918-926, 2012.

14 R. Vitorino, H. Jorge, and L. Neves, "Loss and reliability optimization for power distribution system operation," Electric Power Systems Research, vol. 96, pp. 177 $184,2013$.

15 N. Gupta, A. Swarnkar, and K. Niazi, "Distribution network reconfiguration for power quality and reliability improvement using genetic algorithms," International Journal of Electrical Power \& Energy Systems, vol. 54, pp. 664-671, 2014

16 H. Arasteh, M. S. Sepasian, and V. Vahidinasab, "An aggregated model for coordinated planning and reconfiguration of electric distribution networks," Energy, vol. 94, pp. 786-798, 2016.

17 D.-L. Duan, X.-D. Ling, X.-Y. Wu, and B. Zhong, "Reconfiguration of distribution network for loss reduction and reliability improvement based on an enhanced genetic algorithm," International Journal of Electrical Power Energy Systems, vol. 64 , pp. $88-95,2015$

18 J. E. Mendoza, M. E. Lopez, C. A. C. Coello, and E. A. Lopez, "Microgenetic multiobjective reconfiguration algorithm considering power losses and reliability indices for medium voltage distribution network," IET Generation, Transmission Distribution, vol. 3, no. 9, pp. 825-840, Sep. 2009

19 J. C. López, M. Lavorato, and M. J. Rider, "Optimal reconfiguration of electrical distribution systems considering reliability indices improvement," International Journal of Electrical Power Energy Systems, vol. 78, pp. 837 - 845, 2016.

20 D.-L. Duan, X.-D. Ling, X.-Y. Wu, and B. Zhong, "Reconfiguration of distribution network for loss reduction and reliability improvement based on an enhanced genetic algorithm," International Journal of Electrical Power Energy Systems, vol. 64 , pp. $88-95,2015$.

21 M. R. Narimani, A. A. Vahed, R. Azizipanah-Abarghooee, and M. Javidsharifi, "Enhanced gravitational search algorithm for multi-objective distribution feeder reconfiguration considering reliability, loss and operational cost," IET Generation, Transmission Distribution, vol. 8, no. 1, pp. 55-69, Jan 2014.

22 N. Gupta, A. Swarnkar, and K. Niazi, "Distribution network reconfiguration for power quality and reliability improvement using genetic algorithms," International Journal of Electrical Power Energy Systems, vol. 54, pp. 664 - 671, 2014

23 N. G. Paterakis, A. Mazza, S. F. Santos, O. Erdinç, G. Chicco, A. G. Bakirtzis, and J. P. S. Catalão, "Multi-objective reconfiguration of radial distribution systems using reliability indices," IEEE Transactions on Power Systems, vol. 31, no. 2, pp. 1048-1062, March 2016.

24 H. Shareef, A. Ibrahim, N. Salman, A. Mohamed, and W. L. Ai, "Power quality and reliability enhancement in distribution systems via optimum network reconfiguration by using quantum firefly algorithm," International Journal of Electrical Power Energy Systems, vol. 58, pp. 160-169, 2014.

25 M. Zare, R. Azizipanah-Abarghooee, R. Hooshmand, and M. Malekpour, "Optimal reconfigurattion of distribution systems by considering switch and wind turbine placements to enhance reliability and efficiency," IET Generation, Transmission Distribution, vol. 12, no. 6, pp. 1271-1284, 2018

26 D. Bernardon, A. Mello, L. Pfitscher, L. Canha, A. Abaide, and A. Ferreira, "Realtime reconfiguration of distribution network with distributed generation," Electric Power Systems Research, vol. 107, pp. 59-67, 2014

27 A. Kavousi-Fard and T. Niknam, "Multi-objective stochastic distribution feeder reconfiguration from the reliability point of view," Energy, vol. 64, pp. $342-354$, 2014.

28 A. Kavousi-Fard, T. Niknam, and M. H. Khooban, "Intelligent stochastic framework to solve the reconfiguration problem from the reliability view," IET Science, Measurement Technology, vol. 8, no. 5, pp. 245-259, Sep. 2014

29 S.-A. Ahmadi, "Human behavior-based optimization: a novel metaheuristic approach to solve complex optimization problems," Neural Computing and Applications, vol. 28, no. 1, pp. 233-244, 2017

30 H. B. A. Merlin, "'search for a minimal-loss operating spanning tree configuration in an urban power distribution system," 1975 .

31 A. Fathy, M. El-Arini, and O. El-Baksawy, "An efficient methodology for optimal reconfiguration of electric distribution network considering reliability indices via binary particle swarm gravity search algorithm," Neural Computing and Applications, vol. 30, no. 9, pp. 2843-2858, Nov 2018. [Online]. Available: https://doi.org/10.1007/s00521-017-2877-z

32 J. L. Gross and J. Yellen, Handbook of graph theory. CRC press, 2004.

33 C. A. Warren, R. Ammon, and G. Welch, "A survey of distribution reliability measurement practices in the us," IEEE Transactions on Power Delivery, vol. 14, no. 1, pp. 250-257, 1999.

34 p. $250-257,1999$. placement and sizing based on voltage stability maximization and minimization of power losses," Energy Conversion and Management, vol. 70, pp. 202 - 210, 2013.
35 M. Chakravorty and D. Das, "Voltage stability analysis of radial distribution networks," International Journal of Electrical Power \& Energy Systems, vol. 23, no. 2 , pp. 129-135, 2001

36 D. Das, D. Kothari, and A. Kalam, "Simple and efficient method for load flow solution of radial distribution networks," International Journal of Electrical Power Energy Systems, vol. 17, no. 5, pp. 335 - 346, 1995.

37 M. Moghavvemi and M. Faruque, "Power system security and voltage collapse: a line outage based indicator for prediction," International Journal of Electrical Power Energy Systems, vol. 21, no. 6, pp. 455 - 461, 1999.

38 M. Abdel-Akher, "Voltage stability analysis of unbalanced distribution systems using backward/forward sweep load-flow analysis method with secant predictor," IET Generation, Transmission Distribution, vol. 7, no. 3, pp. 309-317, March 2013.

39 E. Kianmehr, S. Nikkhah, V. Vahidinasab, D. Giaouris, and P. Taylor, "A resiliencebased architecture for joint distributed energy resources allocation and hourly network reconfiguration," IEEE Transactions on Industrial Informatics, 2019.

40 Ji-Pyng Chiou, Chung-Fu Chang, and Ching-Tzong Su, "Variable scaling hybrid differential evolution for solving network reconfiguration of distribution systems," IEEE Transactions on Power Systems, vol. 20, no. 2, pp. 668-674, May 2005.

41 V. Vahidinasab and S. Jadid, "Bayesian neural network model to predict day-ahea electricity prices," European Transactions on Electrical Power, vol. 20, no. 2, pp. 231-246, 2010.

42 A. Baharvandi, J. Aghaei, A. Nikoobakht, T. Niknam, V. Vahidinasab, D. Giaouris, and P. Taylor, "Linearized hybrid stochastic/robust scheduling of active distribution networks encompassing pvs," IEEE Transactions on Smart Grid, 2019.

$43 \mathrm{H}$. Nezamabadi and V. Vahidinasab, "Market bidding strategy of the microgrids considering demand response and energy storage potential flexibilities," IET Generation, Transmission \& Distribution, vol. 13, no. 8, pp. 1346-1357, 2019.

44 B. Jeddi, V. Vahidinasab, P. Ramezanpour, J. Aghaei, M. Shafie-khah, and J. P. Catalão, "Robust optimization framework for dynamic distributed energy resources planning in distribution networks," International Journal of Electrical Power \& Energy Systems, vol. 110, pp. 419-433, 2019.

\section{Appendix}

In order to obtain (16) from (15) and (14) the following steps are required:

$$
\begin{gathered}
I_{i j}=\frac{V_{s}-V_{r}}{r_{i j}+j x_{i j}} \\
I_{i j}=\frac{P_{r}-j Q_{r}}{V^{*}}
\end{gathered}
$$

$$
\frac{\left|V_{s}\right| \angle \delta_{s}-\left|V_{r}\right| \angle \delta_{r}}{r_{i j}+j x_{i j}}=\frac{P_{r}-j Q_{r}}{\left|V_{r}\right| \angle-\delta_{r}}
$$

$$
\left|V_{s}\right|\left|V_{r}\right| \angle\left(\delta_{s}-\delta_{r}\right)-\left|V_{r}\right|^{2}=\left(P_{r}-j Q_{r}\right)\left(r_{i j}+j x_{i j}\right)
$$

$$
\begin{array}{r}
\left(\left|V_{s}\right|\left|V_{r}\right| \cos \left(\delta_{s}-\delta_{r}\right)-\left|V_{r}\right|^{2}\right)+j\left(\left|V_{s}\right|\left|V_{r}\right| \sin \left(\delta_{s}-\delta_{r}\right)\right) \\
=\left(P_{r} r_{i j}+Q_{r} x_{i j}\right)+j\left(P_{r} x_{i j}-Q_{r} r_{i j}\right)
\end{array}
$$

Real and imaginary parts in the both sides of the above equation can be separately equal to each other. Therefore, for real parts:

$$
\left|V_{s}\right|\left|V_{r}\right| \cos \left(\delta_{s}-\delta_{r}\right)=\left|V_{r}\right|^{2}+P_{r} r_{i j}+Q_{r} x_{i j}
$$

\section{For imaginary parts}

$$
\left|V_{s}\right|\left|V_{r}\right| \sin \left(\delta_{s}-\delta_{r}\right)=P_{r} x_{i j}-Q_{r} r_{i j}
$$

By adding the squares of (8.6) and (8.7), and rearranging, the following equation is obtained:

$$
\begin{array}{r}
\left|V_{r}\right|^{4}-\left|V_{s}\right|^{2}\left|V_{r}\right|^{2}+2\left|V_{r}\right|^{2}\left(P_{r} r_{i j}-Q_{r} x_{i j}\right) \\
+\left(P_{r}^{2}+Q_{r}^{2}\right)\left(r_{i j}^{2}+x_{i j}^{2}\right)
\end{array}
$$

The above equation is given in (16)-(18) 